Mini Review

\title{
OXIDATIVE STRESS AND POST-STROKE DEPRESSION
}

\author{
D. Komsiyska* \\ Department of Neurology and Psychiatry, Medical Faculty, Trakia University, \\ Stara Zagora, Bulgaria
}

\begin{abstract}
The topic of post-stroke depression etiology is reviewed in two main approaches. Some suggest that post-stroke depression is caused by the brain damage itself. On the contrary, others assume that this is a psychologic response to injuries or loss. Many discoveries can be examined as evidence for both the physiological and psychosocial mechanism of post-stroke depression. The two methods are not selfexcluding, but instead describe post-stroke depression as a complex and multifactorial disease with interactions between the physiological and environmental factor.

One hypothesis about depression occurrence is the inflammatory, oxidative and nitrosative stress (IO\&NS) depression theory. Oxidative stress mechanisms are implied in the pathogenesis of mental diseases. The brain is considered particularly vulnerable to oxidative damage, due to its relatively high oxygen utilization and thus generation of free radical subordinate products, its modest antioxidant protections and its lipid-rich resistance.
\end{abstract}

Key words: post-stroke depression, free radicals, oxidative stress

\section{INTRODUCTION}

One of the most difficult issues in contemporary clinical practice is the problem about the treatment and rehabilitation of cerebral stroke patients. Depression is common consequence of stroke. Approximately one third of stroke survivors suffer from significant depression symptoms (1). Post-stroke depression could have multifactorial etiology, including organic and reactive components.

Oxidative stress is included in the pathogenesis for a wide variety of disease conditions and can be a common pathogenic mechanism, lying on the basis of many mental disorders, because the brain is relatively more vulnerable to oxidative damage. In the last couple of years oxidative stress is reviewed as one of the contributory factors in depression pathogenesis. Recently, it is also discussed as a concomitant factor in many chronic neurodegenerative pathologies and acute cerebrovascular disorders like stroke.

\footnotetext{
*Correspondence to: Detelina Yordanova Komsiyska, Department of Neurology and Psychiatry, Medical Faculty, Trakia University, Stara Zagora, Bulgaria, e-mail: detelinakomsiiska@abv.bg,tel.: +359898277673/ 042648877
}

\section{OXIDATIVE STRESS}

Violating the balance between processes, that produce ROS /reactive oxygen species/, and antioxidant protective systems leads to oxidative stress occurrence, causing cellular damage and direct inhibition of enzyme proteins (2). Oxidative stress represents an imbalance between the biochemical ROSgenerating processes and the ability of a biological system to neutralize them. As a result, new ROS are formed faster than the cellular defense systems' capability to eliminate them (3). The development of oxidative stress and its consequences depend on the capabilities of the body alone or on outside intervention to restore the physiological balance between pro-oxidants and antioxidants (4).

The theory of oxidative stress as a pathophysiological mechanism can be explained generally with the concept, usually called "oxygen paradox", that while oxygen is essential for aerobic life, large amounts of its free radical metabolic byproducts are toxic (5). Briefly, these free radicals play an integral role in cell signaling, physiological immunological responses and mitoses. However, due to their highly unstable molecules with unpaired electrons, they have different oxidative forces and therefore may potentially damage cellular 
proteins, lipids, carbohydrates and nucleic acids (6). The resulting cell injury may range from cellular structural damage and mitotic blocking to apoptosis and cellular necrosis, depending on the level of severity of the oxidative stress $(7,8)$. The major groups of free radicals in living organisms are reactive oxygen species (ROS) and reactive nitrogen species (RNS), which are respectively collective terms for oxygen- and nitrogenderived radicals, and some non-radicals which are ready to become radicals $(9,10)$.

Oxidative stress mechanisms are implied in the pathogenesis of mental illnesses. This hypothesis has a theoretical appeal, because the brain is considered to be particularly vulnerable to oxidative damage for several reasons. These include its relatively high oxygen utilization and thus generation of free radical byproducts, its modest antioxidant defenses, its lipid-rich stability that delivers ready for oxidation substrates, reduction of potential for some neurotransmitters and the presence of redox catalytic metals such as iron and copper $(9,11)$.

\section{OXIDATIVE STRESS AND STROKES}

In the pathogenesis of acute ischemic stroke low molecular antioxidants are absorbed in the reaction with free radicals generated during oxidative stress. The endogenous catalytic system comprises a number of antioxidant enzymes such as superoxide dismutase, catalase, and glutathione peroxidase. Among these, the first line of defense is the catalase, which decomposes hydrogen peroxide, since its accumulation can lead to the formation of free radicals $(12,13)$.

Oxidative stress culminates due to the imbalance between pro-oxidants and antioxidants and consecutive excessive production of reactive oxygen species. Reactive oxygen species are biphasic, taking part in normal physiological processes, but are also involved in a number of disease processes, which mediate damage to cell structures, including lipid membranes, proteins and DNA. Cerebral circulatory system is the main objective of oxidative stress, because it plays a critical role in the pathogenesis of ischemic brain damage as a result of a cerebrovascular attack. Superoxide, the main reactive oxygen species, and its derivatives cause vasodilatation by opening potassium channels, as well as deterioration of vascular reactivity. However, reactive oxygen species are involved in normal physiological processes, including cell signaling, induction in mitogenesis and immune defense. One study focuses on cellular and vascular aspects of generation of reactive oxygen and nitrogen species and their role in the pathogenesis of the ischemic reperfusion phenomena (14).

Imbalance between cell production of free radicals and the ability of cells to prevent them is one of the leading mechanisms that contribute to neuronal damage (15). These conditions promote oxidative and nitrogen modifications in some amino acids. Proteins are one of the main objectives of reactive oxidative or nitrogen species. Oxidative and nitrogen changes in proteins include the formation of carbonyl groups, oxidation of thiol groups and the generation of nitrotyrosine (16).

The brain is susceptible to secondary damage from oxidative cellular injury or necrosis of the neurotoxic effects of released stimulating amines (mainly glutamate) and iron, and the activated inflammatory response (9). This intrinsic oxidative sensitivity of the brain, along with growing evidence of neurodegenerative changes associated with many psychiatric syndromes suggest that oxidative damage can be a worthy pathogenic candidate.

In one study a statistically significant increased level of carbonyl groups in plasma proteins, reduced levels of plasma protein thiol groups, reduced catalase activity in erythrocytes and an increased level of plasma protein 3-nitrotyrosine were observed in stroke patients respectively to the control group (17). In the case of the carbonyl groups and 3-nitrotyrosine the observed increase in oxidative and nitrative protein damage after stroke is more than twice as high in patients with stroke.

After the beginning of cerebral ischemia oxidative stress plays an important role in inflammatory reactions $(18,19)$. In postischemic brains oxidative stress triggers the activation of microglia and astrocytes (20), leading to sharp increases in the levels of inflammatory mediators such as cytokines, chemokines and matrix metalloproteases (21), and causing a loss of integrity of the endothelial cells in the brain, as manifestated by the upregulation of cell adhesion molecules and neurotrophic infiltration (22). This is likely to provoke a consecutive secondary inflammation and glial immune response, resulting in permanent damage to the brain cells (21).

Redox-mediated increase in free radicals in post-ischemic brains leads to augmented 
expression of several proinflammatory genes, which expression is mediated through a transcription nuclear factor - kappa-B or NF$\kappa B$ (21). Proinflammatory reactions, mediated by $\mathrm{NF}-\kappa \mathrm{B}$ and innate immune responses, are important properties in cerebral ischemic conditions $(23,24,25)$.

\section{ETIOLOGY OF POST-STROKE DEPRESSION}

The pathogenesis of psychopathological conditions, occurring in stroke, is always complex and not sufficiently studied. For many years the main reason for the development of post-stroke depression was considered only a psychogenic response to the emerging disability (26). Subsequently psychosocial factors have begun to attach significant importance of endogenous factors (individual characteristics of the individual and hereditary predisposition).

The mechanisms, responsible for the development of depression, are widely discussed and evidence suggests heterogeneous and mixed etiologies of the disease. A large number of studies provide hypotheses for the development of depression. These assumptions include the monoamine theory of depression with its modifications (27), the hypothesis of a distraught neuroplasticity (28) and the inflammatory, oxidative and nitrosative stress (IO \& NS) theory of depression $(29,30)$. Data suggests that depression is associated with possible increases in the number and activity of macrophages / monocytes with immunocompetent phagocytic cells in the brain, producing free radicals (31). The evidence shows that the depression belongs to a range of neurodegenerative disorders and is accompanied by a reduced antioxidant status and an induction of oxidative and nitrogen pathways. Also there is a significant association between depression and polymorphisms in genes involved in oxidative and nitrogen pathways, such as manganese superoxide dismutase, catalase, and myeloperoxidase (32).

It is assumed that the interruption of the pathways of biogenic amines from stroke, which causes disorders in the synthesis and metabolism of monoamine transmitters, may play an etiologic role in the post-stroke depression $(33,34,35)$. The deficit of the monoamine transmitters dopamine, serotonin and noradrenaline, or the damage to the entire monoamine neurotransmitter system in various brain circuits, can cause depression (36). In a study of both post-stroke depression and endogenous depression interest is shifted from the very monoamine neurotransmitters to their receptors and to the molecular events that trigger these receptors. These include a transduction of stream signal and regulation of gene expression. For example, it is assumed that under stress, the gene for brain-derived neurotrophic factors can be suppressed (36). A new hypothesis for post-stroke depression is an increased production of proinflammatory cytokines (37) and the importance of specific high-risk genes (38). In a study of Cichon et al. 2015 only the oxidative damage of proteins is related to the degree of post-stroke depression, while nitration of proteins does not show such a relationship (17).

\section{BIOMARKERS FOR OXIDATIVE STRESS IN DEPRESSION}

Studies, carried out on post-mortem tissue from depressed patients, showed reduced levels of hippocampus, anatomical structure, involved in anxiety and depressive behaviors, and cortical BDNF (39). Moreover polymorphism in the BDNF gene is associated with depression (40-43). Accumulation of lipofuscin granules has been described in biopsies of patients suffering from major depressive disorder (MDD), indicating the effects of oxidative stress on the neurovascular abnormalities in these patients (44).

\section{CONCLUSION}

Equally strong evidence has implicated oxidative stress in the pathogenesis of poststroke depression. There are several reasons why the brain and the nerves are especially vulnerable to oxidative stress. The brain and nerve tissue contain relatively low level of antioxidants. In addition, those brain regions that are rich in catecholamines are exceptionally vulnerable to free radical generation. The catecholamine adrenaline, noradrenalin, and dopamine can spontaneously break down (auto-oxidise) to free radicals, or can be metabolized to radicals by the endogenous enzymes such as MAO (monoamine oxidases). The pathophysiological relationships between oxidative stress and depression, and the potential benefits of antioxidant supplementation deserve further research.

\section{REFERENCES}

1. Hackett, M., Yapa, C., Parag, V., Anderson, C., Frequency of depression after stroke. A systematic review of observational studies. Stroke, 36: 1330-1340, 2005.

2. Chopra, S., Wallace, H., Induction of spermidine/spermine N1-acetyltransferase in human cancer cells in response to 
increase of reactive oxygen species. Biochem pharmacol, 55: 1119-1123, 1998.

3. Gadzheva, V., Free radicals and oxidative stress,"Oxidative stress, cancer and chemotherapy": 3-19, 2007.

4. Himmelfarb, J. and Hakim, R., Curr Opin Nephrol Hypertens. Oxidative stress in uremia, Curr Opin Nephrol Hypertens., 12 (6): 593-598, 2003.

5. Davies, K., Oxidative stress: the paradox of aerobic life, Biochemical Society Symposium, 61: 1-31, 1995.

6. Filomeni, G., Ciriolo, M., Redox control of apoptosis: an update. Antioxidants and Redox Signaling, 8: 2187-2192, 2006.

7. Davies, K., Oxidative stress, antioxidant defenses, and damage removal, repair, and replacement systems, IUBMB Life, 50: 279-289, 2000.

8. Finkel, T., Holbrook, N., Oxidants, oxidative stress and the biology of ageing, Nature, 408: 239-247, 2000.

9. Halliwell, B., Oxidative stress and neurodegeneration: where are we now?, Journal of Neurochemistry, 97: 1634-1658, 2006.

10.Pacher, P., Beckman, J., Liaudet, L., Nitric oxide and peroxynitrite in health and disease, Physiological Reviews, 87: 315424, 2007.

11.Valko, M., Leibfritz, D., Moncol, J., Cronin, M., Mazur, M., Telser, J., Free radicals and antioxidants in normal physiological functions and human disease, International Journal of Biochemistry and Cell Biology, 39: 44-84, 2007.

12.Cojocaru, I., Cojocaru, M., Sapira, V., Ionescu, A., "Evaluation of oxidative stress in patients with acute ischemicstroke", Romanian Journal of Internal Medicine, 51: 97-106, 2013.

13.Golden, T. and Patel, M., "Catalytic antioxidants and neurodegeneration", Antioxidants \& Redox Signaling, 11: 555569, 2009.

14.Allen, C., Bayraktutan, U., Oxidative stress and its role in the pathogenesis of ischaemic stroke, Int J Stroke, 4: 461-70, 2009.

15.Radak, D., Resanovic, I. and Isenovic, E., "Link between oxidative stress and acute brain ischemia," Angiology, 65: 667-676, 2014.

16.Miller, E., Walczak, A., Saluk, J., Ponczek, M. and Majsterek, I., "Oxidative modification of patient's plasma proteins and its role in pathogenesis of multiple sclerosis", Clinical Biochemistry, 45: 2630, 2012.

17.Cichoń, N., Bijak, M., Miller, E., Niwald, M., Saluk, J., Poststroke depression as a factor adversely affecting the level of oxidative damage to plasma proteins during a brain stroke, Oxid Med Cell Longev., 10: 408-745, 2015.

18.Wang, X. and Lo, E., "Triggers and mediators of hemorrhagic transformation in cerebral ischemia", Molecular Neurobiology, 28: 229-244, 2003.

19.God'inez-Rub'i, M., Rojas-Mayorqu'1n, A., and Ortũno-Sahag'un D., "Nitric oxide donors as neuroprotective agents after an ischemic stroke-related inflammatory reaction", Oxidative Medicine and Cellular Longevity, vol. 2013, Article ID 297357: 1$16,2013$.

20.Love, S., "Oxidative stress in brain ischemia”, Brain Pathology, 9: 119-131, 1999.

21.Lakhan, S., Kirchgessner, A. and Hofer, M., "Inflammatory mechanisms in ischemic stroke: therapeutic approaches," Journal of Translational Medicine, vol. 7, article 97, 2009.

22.Zoppo, G. and Hallenbeck, J., “Advances in the vascular pathophysiology of ischemic stroke", Thrombosis Research, 98: V73V81, 2000.

23.Harari, O. and Liao, J., "NF- $\mathrm{BB}$ and innate immunity in ischemic stroke", Annals of the New York Academy of Sciences, 1207: 3240, 2010.

24.Nichols, T., "NF- $\kappa \mathrm{B}$ and reperfusion injury", Drug News \& Perspectives, 17: 99-104, 2004.

25.Wei, Z., Chigurupati, S., Arumugam, T., Jo, D.-G., Li, H. and Chan, S., "Notch activation enhances the microglia-mediated inflammatory response associated with focal cerebral ischemia", Stroke, 42: 25892594, 2011.

26.Gainotti, G., Azzoni, A., Gasparini, F., Marra, C., Razzano, C., Relation to lesion location to verbal and nonverbal mood measures in stroke patients, Stroke, 28: 2145-2149, 1997.

27.Farvolden, P., Kennedy, S., Lam, R., Recent developments in the psychobiology and pharmacotherapy of depression: optimising existing treatments and novel approaches for the future. Expert Opin Investig Drugs, 12: 65-86, 2003.

28.Christoffel, D., Golden, S., Russo, S., Structural and synaptic plasticity in stressrelated disorders,_Rev Neurosci, 22:535649, 2011.

29.Leonard, B., Maes, M., Mechanistic explanations how cell-mediated immune activation, inflammation and oxidative and nitrosative stress pathways and their sequels and concomitants play a role in the 
pathophysiology of unipolar depression, Neurosci Biobehav Rev, 36: 764-85, 2012.

30.Maes, M., An intriguing and hitherto unexplained co-occurrence: Depression and chronic fatigue syndrome are manifestations of shared inflammatory, oxidative and nitrosative (IO\&NS) pathways,_Prog Neuropsychopharmacol Biol Psychiatry, 35: 784-94, 2011.

31.Maes, M., Depression is an inflammatory disease, but cell-mediated immune activation is the key component of depression, Prog Neuropsychopharmacol Biol Psychiatry, 35: 664-75, 2011.

32.Maes, M., Galecki, P., Chang, Y., Berk, M., "A review on the oxidative and nitrosative stress (O\&NS) pathways in major depression and their possible contribution to the (neuro) degenerative processes in that illness", Progress in Neuro-Psychopharmacology and Biological Psychiatry, 35: 676-692, 2011.

33.Bryer, J., Starkstein, S., Votypka, V., Parikh, R., Price, T., Robinson, R., Reduction of CSF monoamine metabolites in posstroke depression: a preliminary report, The Journal of Neuropsychiatry and Clinical Neurosciences, 4: 440-442, 1992.

34.Ramasubbu, R., Flint, A., Brown, G., Awad, G., Kennedy, S., A neuroendocrine study of serotonin function in depressed stroke patients compared to non depressed stroke patients and healthy controls, Journal of Affective Disorders, 52,121-133, 1999.

35.Rocco, A., Afra, J., Toscano, M., Sirimarco, G., Di Clemente, L., Altieri, M., Acute subcorticalstroke and serotonergic modification: a IDAP study, European Journal of Neurology, 14: 1378-1382, 2007.

36.Stahl, S., Depression and Bipolar Disorder. Stahl's Essential Psychopharmacology (3rd ed.), Cambridge University Press, 2008.
37. Spalletta, G., Boss, P., Ciaramella, A., Bria, P., Caltagirone, C., Robinson, R. The etiology of poststroke depression: a review of the literature and a new hypothesis involving inflammatory cytokines, Molecular Psychiatry, 11: 984-991, 2006.

38. Ramasubbu, R., Tobias, R., Bech-Hansen, N., Extended evaluation of serotonin transporter gene functional polymorphisms in subject with post-stroke depression, Canadian Journal of Pscyhiatry, 53: 197201, 2008.

39.Dwivedi, Y., Rizavi, H., Conley, R., Roberts, R., Tamminga, C., Pandey, G., "Altered gene expression of brainderived neurotrophic factor and receptor tyrosine kinase B in postmortem brain of suicide subjects", Archives of General Psychiatry, 60: 804-815, 2003.

40.Sklar, P., Gabriel, S., McInnis, M., "Family-based association study of 76 candidate genes in bipolar disorder: BDNF is a potential risk locus", Molecular Psychiatry, 7: 579-593, 2002.

41.Sen, S., Nesse, R., Stoltenberg, S., "ABDNF coding variant is associated with the NEO personality inventory domain neuroticism, a risk factor for depression", Neuropsychopharmacology, 28: 397-401, 2003.

42.Kaufman, J., Yang, B.-Z., DouglasPalumberi, H., "Brainderived neurotrophic factor-5-HTTLPR gene interactions and environmental modifiers of depression in children", Biological Psychiatry, 59: 673680, 2006.

43.Chen Z.-Y., Jing D., Bath K., "Genetic variant BDNF (Val66Met) polymorphism alters anxiety-related behavior", Science, 314: 140-143, 2006.

44.Najjar, S., Pearlman, D., Hirsch, S., "Brain biopsy findings link major depressive disorder to neuroinflammation, oxidative stress, and neurovascular dysfunction: a case report”, Biological Psychiatry, 2013. 